\title{
Efficacy of computed tomography features in predicting stage III thymic tumors
}

\author{
YAN SHEN $^{1,2^{*}}$, JIANDING YE $^{2 *}$, WENTAO FANG $^{3}$, YU ZHANG $^{4}$, XIAODAN YE ${ }^{2}$, \\ YONGHONG MA ${ }^{2}$, LIBO $\mathrm{CHEN}^{5}$ and MINGHUA LI ${ }^{1}$
}

\begin{abstract}
${ }^{1}$ Institute of Diagnostic and Interventional Radiology, Shanghai Jiao Tong University Affiliated Sixth People's Hospital, Shanghai 200233; Departments of ${ }^{2}$ Radiology and ${ }^{3}$ Thoracic Surgery, Shanghai Chest Hospital, Shanghai Jiao Tong University, Shanghai 200030; ' Department of Radiology, Shanghai Jinshan Hospital, Fudan University, Shanghai 201508;

${ }^{5}$ Department of Nuclear Medicine, Shanghai Jiao Tong University Affiliated Sixth People's Hospital, Shanghai 200233, P.R. China
\end{abstract}

Received April 1, 2015; Accepted May 13, 2016

DOI: $10.3892 / \mathrm{ol} .2016 .5429$

\begin{abstract}
Accurate assessment of the invasion of intrathoracic structures by stage III thymic tumors assists their appropriate management. The present study aimed to evaluate the efficacy of computed tomography (CT) features for the prediction of stage III thymoma invasion. The pre-operative CT images of 66 patients with confirmed stage III thymic tumors were reviewed retrospectively. The $\mathrm{CT}$ features of invasion into the mediastinal pleura, lungs, pericardium and great vessels were analyzed, and their sensitivity, specificity, positive predictive value (PPV), negative predictive value and accuracy were calculated. For mediastinal pleural and pericardial invasion, an absence of space between the tumor and the mediastinal pleura/pericardium with mediastinal pleural/pericardial thickening and pleural/pericardial effusion exhibited a specificity and PPV of $100 \%$, respectively. For lung invasion, a multi-lobular tumor convex to the lung with adjacent lung abnormalities exhibited a specificity and PPV of 91.2 and $81.3 \%$, respectively. For vessel invasion, the specificity and PPV were each $100 \%$ for tumors abutting $\geq 50 \%$ of the vessel circumference, and for tumor oppression, deformation and occlusion of the vessel. In conclusion, recognition of the appropriate CT features can serve as a guide to invasion by stage III thymic tumors, and can facilitate the selection of appropriate pre-operative treatment.
\end{abstract}

Correspondence to: Dr Minghua Li, Institute of Diagnostic and Interventional Radiology, Shanghai Jiao Tong University Affiliated Sixth People's Hospital, 600 Yishan Road, Shanghai 200233, P.R. China

E-mail: liminghua1952@126.com

${ }^{*}$ Contributed equally

Key words: computed tomography, feature, thymic tumor, stage, invasion

\section{Introduction}

Thymic tumors, including thymomas and thymic carcinomas, are the most common primary neoplasms of the anterior mediastinum, with one study quoting an incidence of 2.5-3.2 per million individuals in the Netherlands (1). Thymic tumors should be considered malignant sue to their tendency for local invasion, pleural dissemination and systemic metastases. In total, 30-60\% of thymic tumors show various degrees of invasion into mediastinal fat and adjacent structures (2-5).

Based on the gross and microscopic invasive properties of the tumors at surgery, the International Thymic Malignancy Interest Group (ITMIG) has adopted the Masaoka-Koga staging system as the official staging system for thymic tumors (6-8). Usually, a complete resection can be achieved by surgery in patients at stage I or II. However, for stage III or IV tumors, particularly where there is locoregional spread into neighboring mediastinal organs (stage III tumors), neoadjuvant therapy provides a survival advantage (9-12). With recent advances in minimally invasive surgical techniques, certain patients with stage III thymic tumors have become eligible for surgical evaluation, but others will require neoadjuvant therapy prior to complete resection (13). In certain cases, patients who have undergone invasive procedures have ultimately been found to have an early-stage thymic tumor; while in others, the pre-operative stage of the tumor has been underestimated, resulting in surgical difficulties (14). Therefore, accurate assessment of tumor invasion into intrathoracic structures is of great value in deciding upon the appropriate management of stage III thymic tumors, and precise staging is important prior to making treatment decisions (15).

At present, computed tomography (CT) is the imaging modality of choice for the evaluation of mediastinal masses. Although there have been several studies focusing on the association between the Masaoka-Koga stage, CT findings and World Health Organization (WHO) histology (16-26), none of these studies were dedicated to an evaluation of CT features for predicting pathological staging. The present study was designed to evaluate the efficacy of CT features in predicting invasion by stage III thymic tumors and to assess whether the features can serve as a guide to selecting the appropriate therapeutic strategies. 


\section{Patients and methods}

Approval and consent. This retrospective study was approved by the Research Committees of the Shanghai Chest Hospital, Shanghai Jiao Tong University (Shanghai, China) and the Shanghai Jiao Tong University Affiliated Shanghai Sixth People's Hospital (Shanghai, China), and a waiver was obtained for informed consent. The study took place between July 2009 and June 2013.

Patient inclusion and exclusion criteria. Patients with pre-operative thymic tumors that had been confirmed by surgical resection and histological examination were enrolled in the present study. All patients were diagnosed with stage III thymic tumors on the basis of invasion into neighboring organs (i.e., the mediastinal pleura, lungs, pericardium or great vessels), as determined by surgical findings and histology. CT imaging was performed in all patients within the 2 weeks prior to surgery. All patients underwent complete tumor resection without pre-operative neoadjuvant therapy. Patients with incomplete data were excluded.

A total of 66 patients (44 males and 22 females) ranging in age from 18-77 years (median, 56 years) were enrolled. Of these, 9 patients presented with myasthenia gravis (MG), 34 with chest pain and a cough, and 7 with dyspnea, while 16 were asymptomatic.

Diagnostic criteria. The tumor stage was classified according to the ITMIG definition of details of the Masaoka-Koga staging system (27). All histopathological specimens were reviewed by an experienced pathologist according to the latest WHO classification (11). The classification included 7 subtypes of thymomas (types A, AB, B1, B1+B2, B2, B2+B3 and $\mathrm{B} 3$ ) and thymic carcinoma, which were determined in accordance with the morphology of epithelial cells and the lymphocyte-to-epithelial cell ratio (28).

Definition of stage III. According to the ITMIG definition of details of the Masaoka-Koga staging system (27), macroscopic or microscopic invasions into neighboring organs (i.e., the pericardium, great vessels or lungs) and any involvement (either partial or penetrating) of the mediastinal pleura were classified as stage III.

CT imaging. The $\mathrm{CT}$ examinations were performed using a Philips Brilliance 64-slice helical CT (Philips Brilliance, Cleveland, Ohio, USA). In all patients, the CT examinations used $64 \times 0.625-\mathrm{mm}$ collimation, a slice thickness of $5.00 \mathrm{~mm}$, a slice increment of $5.00 \mathrm{~mm}$, a pitch of $1.08,120 \mathrm{kVp}$ and $200 \mathrm{~mA}$, and a 512x512 image matrix through the thorax prior to and after intravenous injection of a contrast medium (iohexol; $300 \mathrm{mg}$ iodine $/ \mathrm{ml} ; 1.5 \mathrm{ml} / \mathrm{kg}$ ) at a rate of $3.0 \mathrm{ml} / \mathrm{sec}$. All CT images were captured at mediastinal (window width, 400-450 HU; window level, 30-50 HU) and lung (window width, 1,000-1,500 HU; window level, -650 to $-500 \mathrm{HU}$ ) windows.

Image analysis. The $\mathrm{CT}$ features of the thymic tumors were reviewed independently by two radiologists with 15 and 8 years of experience, respectively. The radiologists were blinded to each other, and to the clinical and pathological information. The radiologist with 15 years of experience reassessed the CT findings 1 month after the initial evaluation. The following anatomical and invasive characteristics were assessed: i) Tumor size: Long diameters were measured in the maximal cross-section CT images. ii) Tumor contours: Smooth, lobulated or irregular. iii) Tumor density: Homogeneous or heterogeneous. The heterogeneous density included gross unevenness and a CT value difference of $20 \mathrm{HU}$ following administration of contrast medium. iv) Calcification: Yes or no. v) Necrosis/cystic components: Yes or no. A non-enhanced region with water density was regarded as either cystic or necrotic. vi) Invasion: The grading according to invasion, as assessed by CT features, is summarized in Table I. Any one of the findings listed in Table I suggested invasion into the organ.

Statistical analysis. Data were expressed as the mean \pm standard deviation for continuous variables and numbers with percentages for categorical variables. A P-value of $<0.05$ was interpreted as a statistically significant difference. The consistency of the assessments by the two radiologists and for the same radiologist prior to and after 1 month was assessed by a weighted $\kappa$ coefficient. The diagnostic efficacy of the CT features in comparison with pathological findings was evaluated by their sensitivity, specificity, positive predictive value (PPV), negative predictive value (NPV) and accuracy. For the statistical analyses, SPSS software version 16.0 (SPSS Inc., Chicago, IL, USA) was used.

\section{Results}

Patients and disease characteristics. Among the 66 patients, all the thymic tumors were located in the anterior mediastinum; on the left side in 31 patients, on the right side in 27 patients and on the midline in 8 patients. The mean size was $5.6 \pm 1.4 \mathrm{~cm}$, ranging from $2.9-9.9 \mathrm{~cm}$. On the basis of the WHO classification, thymic carcinomas were present in 25 patients $(25 / 66,37.9 \%)$, type B3 thymomas in $18(18 / 66,27.3 \%)$, type $\mathrm{B} 2+\mathrm{B} 3$ thymomas in $8(8 / 66,12.1 \%)$, type $\mathrm{B} 2$ thymomas in $10(10 / 66,15.2 \%)$, type $\mathrm{B} 1+\mathrm{B} 2$ thymomas in $3(3 / 66,4.5 \%)$ and type A thymomas in $2(2 / 66,3.0 \%)$. The pathological results for the 66 patients were as follows: Single organ invasion including pleural invasion in 5 cases and pericardial invasion in 18 cases; pleural and lung invasion in 9 cases; pleural and pericardial invasion in 6 cases; pericardial and vessel invasion in 2 cases; pleural, lung and pericardial invasion in 15 cases; pleural, pericardial and vessel invasion in 3 cases; and pleural, lung, pericardial and vessel invasion in 8 cases. Overall, there was pleural invasion in 46 cases $(46 / 66,69.7 \%)$, lung invasion in $32(32 / 66,48.5 \%)$, pericardial invasion in $52(52 / 66,78.8 \%)$ and vessel invasion in 13 (13/66, 19.7\%).

The $\kappa$ values ranged from $0.74-0.93$, suggesting good agreement between the two radiologists, and prior to and after 1 month. The weighted $\kappa$ coefficients for the two radiologists, and for the same radiologist prior to and after 1 month are shown in Table II.

CT features. On CT, the thymic tumors presented as oval-shaped in 14 patients $(21.2 \%)$ and as an lobulated or irregular anterior mediastinal mass in 52 patients (78.8\%). 


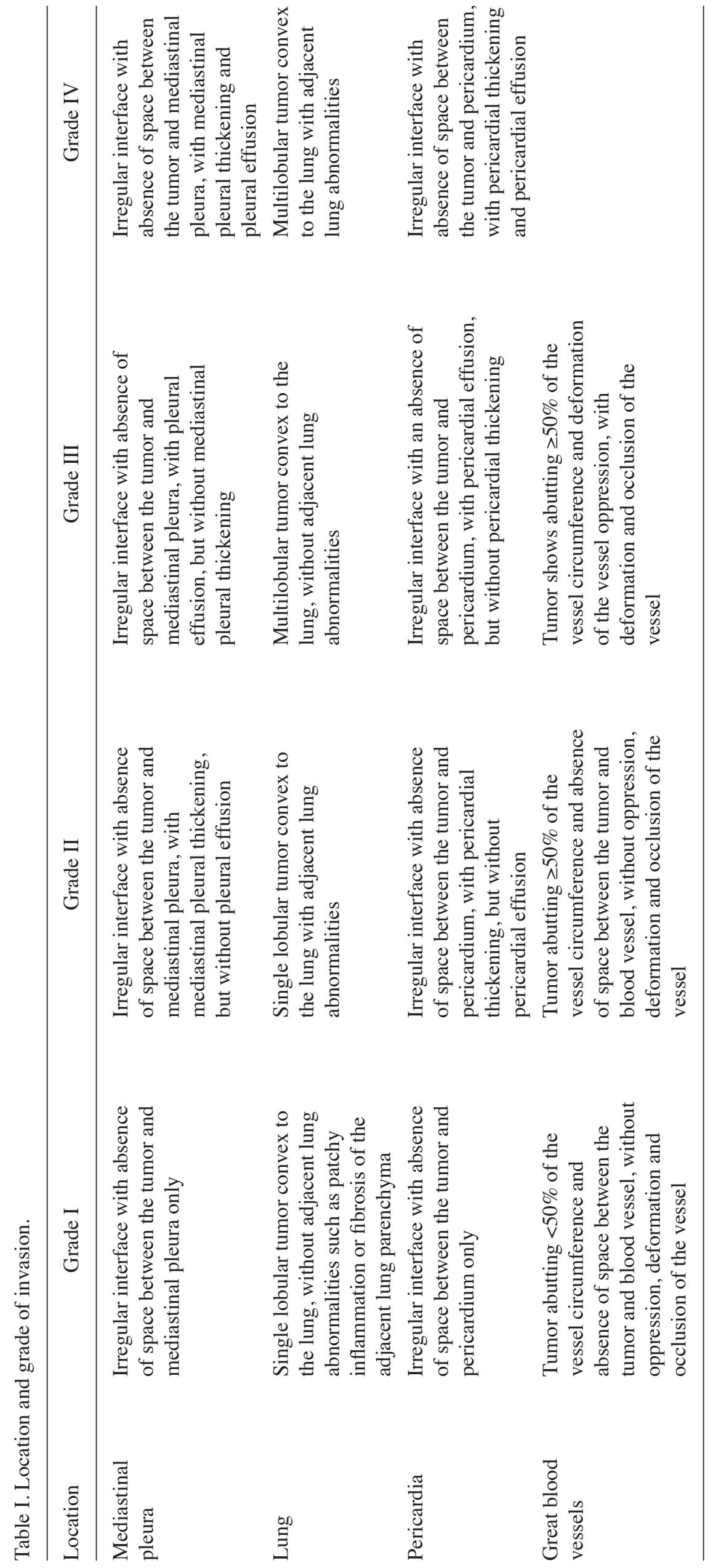


Table II. Consistency estimates of the radiologist evaluations.

$\kappa$ value

$\begin{array}{lll}\text { Mediastinal invasion } & \text { Grade } & \text { Of the same radiologist }\end{array}$

Pleural invasion

$\begin{array}{ccc}\text { I } & 0.78 & 0.78 \\ \text { II } & 0.89 & 0.81 \\ \text { III } & 0.78 & 0.78 \\ \text { IV } & 0.79 & 0.79\end{array}$

Lung invasion

$\begin{array}{lll}\text { I } & 0.89 & 0.89 \\ \text { II } & 0.92 & 0.82 \\ \text { III } & 0.93 & 0.82 \\ \text { IV } & 0.92 & 0.84\end{array}$

Pericardial invasion

$\begin{array}{ccc}\text { I } & 0.85 & 0.82 \\ \text { II } & 0.84 & 0.80 \\ \text { III } & 0.84 & 0.77 \\ \text { IV } & 0.88 & 0.83\end{array}$

Vessel invasion

$\begin{array}{ccc}\text { I } & 0.88 & 0.83 \\ \text { II } & 0.78 & 0.78 \\ \text { III } & 0.85 & 0.74\end{array}$

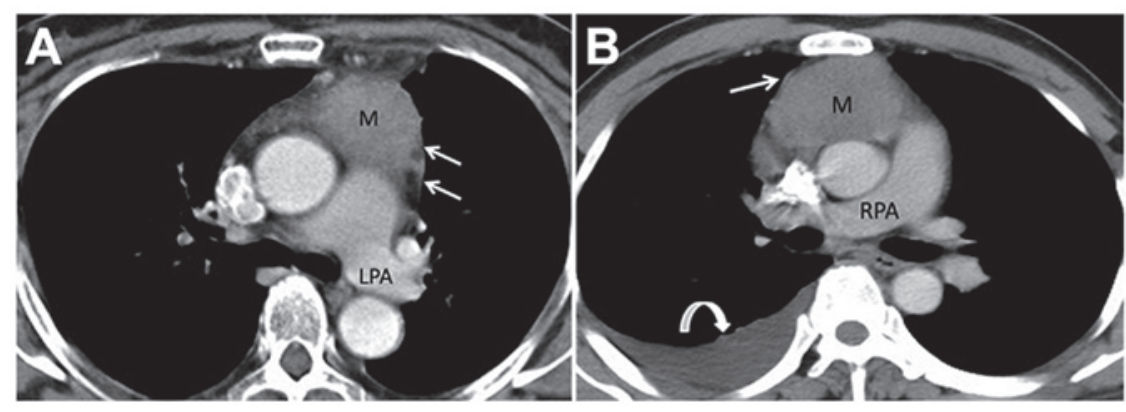

Figure 1.Examples of mediastinal pleural invasion.(A) A 56-year-old woman with a stage III thymic carcinoma (mediastinal pleural invasion). Contrast-enhanced chest computed tomography (CT) at the level of the left pulmonary artery (LPA) demonstrates a left anterior mediastinal mass (M) with an absence of space between the tumor and the mediastinal pleura, with mediastinal pleural thickening (straight arrows). (B) A 58-year-old man with a type B3 thymoma at stage III (mediastinal pleural invasion). Contrast-enhanced chest CT at the level of the right pulmonary artery (RPA) demonstrates a right anterior mediastinal mass (M) with absence of space between the tumor and the mediastinal pleura, with mediastinal pleural thickening (straight arrow) and right pleural effusion (curved arrow). The invasion was confirmed by the same experienced pathologist.

A heterogeneous density was exhibited in 51 patients $(77.3 \%)$, 40 of whom (78.4\%) exhibited necrotic and/or cystic components, and 19 of whom (37.3\%) showed calcification.

Examples of CT images from patients with mediastinal pleural invasion are shown in Fig. 1. The manifestations of the tumor and the mediastinal pleura are shown in Table II. The majority of patients $(39 / 66,59.1 \%)$ had an irregular interface with an absence of space between the tumor and mediastinal pleura.

Examples of CT images from patients with lung invasion are shown in Fig. 2. Thymic tumors most often $(21 / 66,31.8 \%)$ presented as a single lobular mass convex to the lung, without adjacent lung abnormalities. There were 20 cases $(30.3 \%)$ of a multilobular mass convex to the lung, without adjacent lung abnormalities.

Examples of CT images from patients with pericardial invasion are shown in Fig. 3. Of the $66 \mathrm{CT}$ images, the most common, in $32(48.5 \%)$ patients, showed an irregular interface with an absence of space between the tumor and the pericardium.

Examples of CT images from patients with great vessel invasion are shown in Fig. 4. This was observed in 18 of the 66 CT images (27.3\%).

Diagnostic efficacy. The diagnostic efficacies of the CT features in predicting stage III tumors are shown in Table III. 


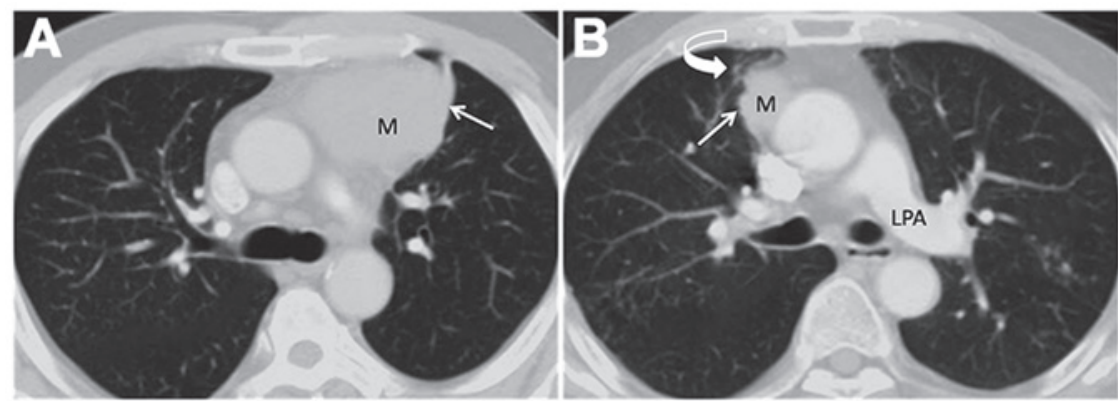

Figure 2. Examples of lung invasion. (A) A 53-year-old man with a stage III thymic carcinoma (left lung invasion). Contrast-enhanced chest computed tomography (CT) at the level of the carina of the trachea showing a left anterior mediastinal mass (M) with a single lobular shape convex to the superior lobe of the left lung, with adjacent lung parenchyma compression (straight arrow). (B) A 75-year-old man with a stage III thymic carcinoma (right lung invasion). Contrast-enhanced chest CT at the level of the left pulmonary artery (LPA) showing a right anterior mediastinal mass (M) with a multilobular shape convex to the superior lobe of the right lung (curved arrow), with patchy inflammation of the adjacent lung parenchyma (straight arrow). The invasion was confirmed by the same experienced pathologist.
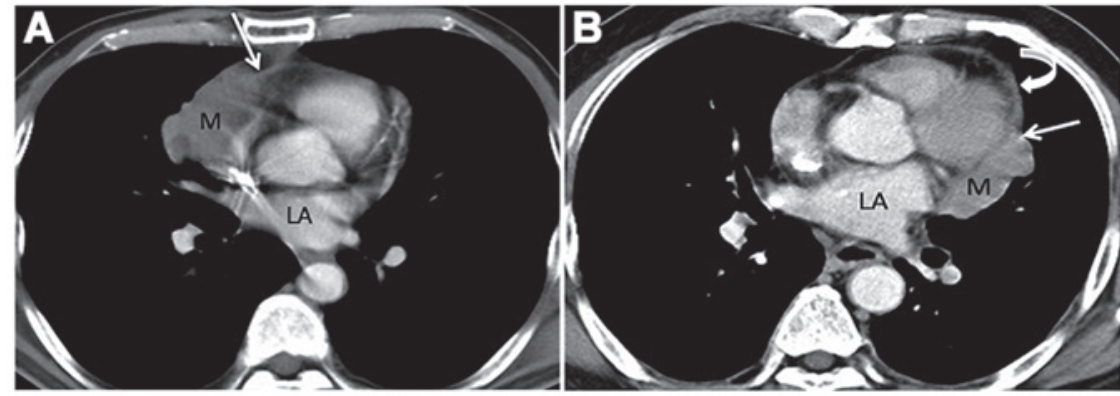

Figure 3. Examples of pericardial invasion. (A) A 57-year-old man with a type B2 thymoma at stage III (pericardial invasion). Contrast-enhanced chest computed tomography (CT) at the level of the left atrium (LA) shows a right anterior mediastinal mass (M) with an absence of space between the tumor and the pericardium, with pericardial thickening (straight arrow). (B) A 55-year-old man with a type B3 thymoma at stage III (pericardial invasion). Contrast-enhanced chest CT at the level of the left atrium (LA) shows a left anterior mediastinal mass (M) with an absence of space between the tumor and the pericardium (straight arrow), with pericardial thickening and pericardial effusion (curved arrow). The invasion was confirmed by the same experienced pathologist.
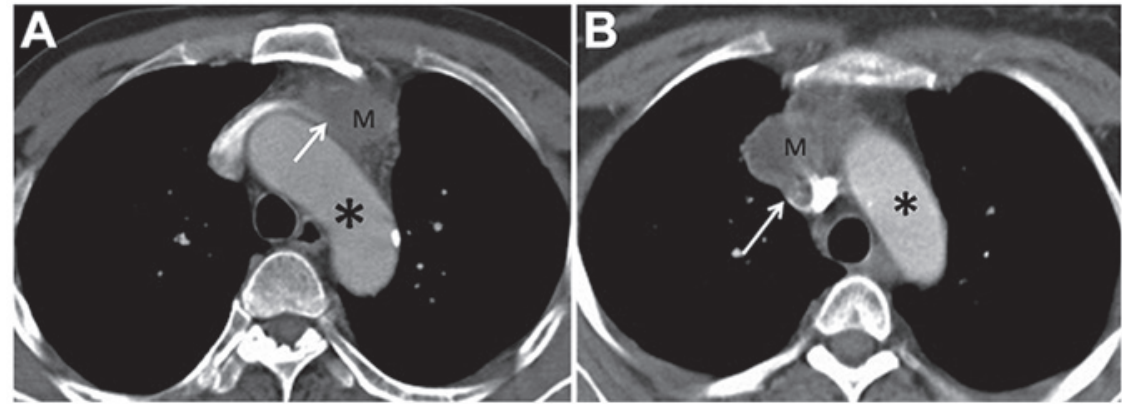

Figure 4. Examples of great vessel invasion. (A) A 62-year-old man with a type B3 thymoma at stage III (left brachiocephalic vein invasion). Contrast-enhanced chest computed tomography (CT) at the level of the aortic arch $(*)$ shows a left anterior mediastinal mass $(\mathrm{M})$ with an absence of space between the tumor and the left brachiocephalic vein (straight arrow). (B) A 41-year-old woman with a type B2+B3 thymoma at stage III (superior vena cava invasion). Contrast-enhanced chest $\mathrm{CT}$ at the level of the aortic arch $(*)$ shows a right anterior mediastinal mass $(\mathrm{M})$ with deformation and partial occlusion of the superior vena cava (straight arrow). The invasion was confirmed by the same experienced pathologist.

Mediastinal pleural invasion was diagnosed in 65 out of the $66 \mathrm{CT}$ images and was confirmed by the pathology results in 46 cases. The specificity and PPV were each $100 \%$ for grade IV.

Lung invasion was diagnosed in 64 out of the $66 \mathrm{CT}$ lung images and was confirmed by the pathology results in 32 cases. The specificity and PPV were 91.2 and $81.3 \%$, respectively, for grade IV.
Pericardial invasion was diagnosed in 61 out of the $66 \mathrm{CT}$ images and was confirmed by the pathology results in 52 cases. For grades II and IV, the specificity and PPV were each $100 \%$.

Great vessel invasion was diagnosed in 18 of the $66 \mathrm{CT}$ images and was verified by the pathology results in 13 cases. For grades II and III, the specificity and PPV were each $100 \%$. 
Table III. Diagnostic efficacy of CT features.

\begin{tabular}{|c|c|c|c|c|c|c|c|}
\hline Site of invasion & $\begin{array}{c}\text { Invasion on } \\
\mathrm{CT}, \mathrm{n}\end{array}$ & $\begin{array}{l}\text { Invasion on } \\
\text { pathology, n }\end{array}$ & Sensitivity, $\%$ & Specificity, \% & PPV, \% & NPV, \% & Accuracy, \% \\
\hline \multicolumn{8}{|l|}{ Pleura } \\
\hline Grade I & 39 & 26 & 56.5 & 35.0 & 66.7 & 25.9 & 50.0 \\
\hline Grade II & 19 & 15 & 32.6 & 80.0 & 79.0 & 34.0 & 47.0 \\
\hline Grade III & 5 & 3 & 6.5 & 90.0 & 60.0 & 29.5 & 31.8 \\
\hline Grade IV & 2 & 2 & 4.4 & 100.0 & 100.0 & 31.3 & 33.3 \\
\hline \multicolumn{8}{|l|}{ Lung } \\
\hline Grade I & 21 & 2 & 6.3 & 44.1 & 9.5 & 33.3 & 25.8 \\
\hline Grade II & 7 & 4 & 12.5 & 91.2 & 57.1 & 52.5 & 50.0 \\
\hline Grade III & 20 & 13 & 40.0 & 79.4 & 65.0 & 58.7 & 60.6 \\
\hline Grade IV & 16 & 13 & 40.6 & 91.2 & 81.3 & 62.0 & 66.7 \\
\hline \multicolumn{8}{|l|}{ Pericardium } \\
\hline Grade I & 32 & 24 & 46.2 & 42.9 & 75.0 & 17.7 & 45.5 \\
\hline Grade II & 12 & 12 & 23.1 & 100.0 & 100.0 & 25.9 & 39.4 \\
\hline Grade III & 7 & 6 & 11.5 & 92.9 & 85.7 & 22.0 & 28.8 \\
\hline Grade IV & 10 & 10 & 19.2 & 100.0 & 100.0 & 25.0 & 36.4 \\
\hline \multicolumn{8}{|l|}{ Great vessels } \\
\hline Grade I & 10 & 5 & 38.5 & 90.6 & 50.0 & 85.7 & 80.3 \\
\hline Grade II & 5 & 5 & 38.5 & 100.0 & 100.0 & 86.9 & 87.9 \\
\hline Grade III & 3 & 3 & 23.1 & 100.0 & 100.0 & 84.1 & 84.9 \\
\hline
\end{tabular}

CT, computed tomography; NPV, negative predictive value; PPV, positive predictive value.

\section{Discussion}

The present study has illustrated pictorially the CT features of stage III thymic tumors. A focus was placed upon the association between the tumors and adjacent structures, such as the mediastinal pleura, lung, pericardium and great vessels. These $\mathrm{CT}$ features proved useful for the identification of tumor invasion for the radiologists and surgeons.

Studies have used CT for differentiating early (stage I and II) from advanced tumors (stage III and IV), according to the surgical classification of stages proposed by Masaoka-Koga (23-25). Preoperative CT features, such as large tumor size, lobulated tumor and infiltration of fat surrounding the tumor, suggest a high probability of Masaoka stage III or IV disease. However, it is challenging to accurately identify the stage of thymoma by the varying degree of fat obliteration between tumor and mediastinal structures $(23,25)$. Proper management decisions for thymic tumors are based largely on the invasion site and the extent to which the tumors are present on CT imaging. It is key to determine to what extent the CT features agree with the pathological results. To the best of our knowledge, this is the first study that has focused on evaluating the efficacy of CT features in predicting stage III thymoma invasion. In the study, the most frequent invasive site was the pericardium, followed by the pleura, lungs and great vessels. The degree of invasion varied according to the association between the tumor and adjacent structures, and identifying true and false phenomena in the $\mathrm{CT}$ images proved to be challenging.
A previous study into the use of pleural invasion features to judge the tumor stage did not demonstrate the diagnostic accuracy of CT findings (25). In the present study, the signs of pleural invasion were subdivided into 4 grades to evaluate the efficacy of CT features in predicting stage III thymoma invasion. It was found that images suggesting an irregular interface with an absence of space between the tumor and pleura was the most invasive sign, and that this exhibited a higher sensitivity $(56.52 \%)$ than the other signs evaluated. However, its PPV was just $66.67 \%$. When the fat space disappeared and mediastinal pleural thickening or pleural effusion occurred simultaneously, the diagnostic specificity was as high as 80.00 and $90.00 \%$, respectively. If all 3 signs occurred at the same time (grade IV), the specificity and PPV each reached $100 \%$. Thus, as long as the absence of a fat space occurred with pleural thickening and/or pleural effusion, mediastinal pleural invasion can be confidently predicted. By contrast, mediastinal pleural invasion does not occur pathologically without these signs. In the present study, the sensitivity, NPV and accuracy of CT features for diagnosing mediastinal pleural invasion ranged from $4.35-56.52 \%$, from $25.93-34.04 \%$ and from $31.82-50.00 \%$, respectively. In the study by Tomiyama et al (24), infiltration of the pleura was identified by CT in only 5 out of 24 cases with surgically proven pleural infiltration. Although it was occasionally difficult to define mediastinal pleural invasion in the present patients, any attempt to focus more consistently on the mediastinal pleura represents a step forward (29). It has been reported that the results obtained using analyses of thinner or thicker sections 
are not significantly different (24); therefore, further research, including magnetic resonance imaging analysis, is required to improve the diagnostic efficacy.

With the exception of a single lobular mass convex to the lung without adjacent lung abnormalities, the diagnostic specificities for the other 3 grades of CT lung invasion features were $91.18 \%$ for grade II, $79.41 \%$ for grade III and $91.18 \%$ for grade IV. Therefore, no lung invasion can be predicted in the absence of these CT characteristics. Since the specificity and PPV were 91.18 and $81.25 \%$, respectively, in grade IV, a multilobular thymic tumor with adjacent lung abnormalities is the most important sign for diagnosing lung invasion via CT imaging. Lung invasion occurred more frequently in multilobular thymic tumors than in single lobular tumors in the present study. The 64 cases of lung involvement based on CT were verified pathologically in 32 patients. In a study by Zerhouni et al (30), in which the CT findings of 10 cases of invasive thymoma were reported, lung involvement was correctly diagnosed by CT in 6 patients. Tomiyama et al (24) reported that infiltration of the adjacent lung was identified by CT in only 1 out of 9 cases with histologically proven pulmonary infiltration. This difference in CT findings compared with the present study was presumably due to the different disease stages.

The identification of pericardial invasion is also an essential step prior to surgery, as it may affect the therapeutic strategy. In grades II and IV in the present study, all cases of pericardial invasion identified by $\mathrm{CT}$ were confirmed by the pathology results, and the diagnostic specificity and PPV were each $100 \%$. As long as there was an absence of a fat space, with pericardial thickening with or without pericardial effusion on CT imaging, pericardial invasion could be confirmed pre-operatively. Since the diagnostic specificity and PPV for grade III were 92.86 and $85.71 \%$, respectively, the CT characteristics of an absence of space between the thymic tumor and pericardium with pericardial effusion are useful in predicting pericardial invasion.

It has been reported that thymic carcinomas have a higher prevalence of great vessel invasion than thymomas $(13,17)$. By contrast, the present study found that invasive thymomas $(61.5 \% ; 8 / 13)$ had a higher prevalence of great vessel invasion than thymic carcinomas $(30.8 \%, 4 / 13)$. The exclusion of inoperative cases in the present study may have contributed to this finding. The diagnostic specificities and PPVs for CT findings in grades II and III were $100 \%$. The CT manifestations of tumors abutting $\geq 50 \%$ of the vessel circumference or oppression, deformation and occlusion of the vessels definitely suggested vessel invasion. Since the specificity for just an absent fat space (grade I) was $90.57 \%$, it can be confidently predicted that no vessel invasion is present if there is space between the tumor and blood vessels. The NPVs and diagnostic accuracies for the other 2 grades ranged from $84.13-86.89 \%$ and from $84.85-87.88 \%$, respectively. Therefore, a lack of invasion can be confidently diagnosed in the absence of these CT features. However, the diagnostic sensitivities for all 3 grades were as low as $23.08-38.46 \%$. Possible reasons for this were patient selection bias and the incomplete resection of certain invaded vessels, which would have resulted in negative pathology findings.

The present study has certain limitations that should be considered alongside the results. The study was retrospective; therefore, there was no way of comparing the predictive value of CT features with any other method of evaluating stage III thymic tumors prior to treatment. Also, the study sample size was quite small due to the limited number of patients; multi-center studies including more patients would provide more evidence for these results, as well as providing evidence of the reproducibility of the CT grading of the thymic tumor features.

In summary, in the first study to focus on evaluating the efficacy of CT features in predicting stage III thymoma, CT features were found to be effective. Consequently, familiarity with CT features for predicting stage III thymic tumors facilitates diagnostic accuracy, and thus may be useful in optimizing patient treatment.

\section{Acknowledgements}

The authors are grateful to Dr Li Zhu, Dr Qun-Hui Chen, Dr Zhi-Chun Zheng, Dr Yi-Feng Jiang (radiologists at the Shanghai Chest Hospital, Shanghai Jiao Tong University, Shanghai, China) and Dr Zhi-Tao Gu (thoracic surgeon at the Shanghai Chest Hospital) for collecting the majority of the imaging data. The authors would like to thank Dr Lei Zhu (pathologist at the Shanghai Chest Hospital) for performing the pathological examinations. This study was partially sponsored by the National Natural Science Foundation of China (grant no. 81271609) and the Shanghai Municipal Commission of Health and Family Planning Scientific Research Task (grant no. 201440442).

\section{References}

1. de Jong WK, Blaauwgeers JL, Schaapveld M, Timens W, Klinkenberg TJ and Groen HJ: Thymic epithelial tumours: A population-based study of the incidence, diagnostic procedures and therapy. Eur J Cancer 44: 123-130, 2008.

2. Batata MA, Martini N, Huvos AG, Aguilar RI and Beattie EJ Jr: Thymomas: Clinicopathologic features, therapy, and prognosis. Cancer 34: 389-396, 1974.

3. Hofmann W, Möller P, Manke HG and Otto HF: Thymoma. A clinicopathologic study of 98 cases with special reference to three unusual cases. Pathol Res Pract 179: 337-353, 1985.

4. Lewis JE, Wick MR, Scheithauer BW, Bernatz PE and Taylor WF: Thymoma. A clinicopathologic review. Cancer 60: 2727-2743, 1987.

5. Salyer WR and Eggleston JC: Thymoma: A clinical and pathological study of 65 cases. Cancer 37: 229-249, 1976.

6. Koga K, Matsuno Y, Noguchi M, Mukai K, Asamura H, Goya T and Shimosato Y: A review of 79 thymomas: Modification of staging system and reappraisal of conventional division into invasive and non-invasive thymoma. Pathol Int 44: 359-367, 1994.

7. Falkson CB, Bezjak A, Darling G, Gregg R, Malthaner R, Maziak DE, Yu E, Smith CA, McNair S, Ung YC, et al: The management of thymoma: A systematic review and practice guideline. J Thorac Oncol 4: 911-919, 2009.

8. Huang J, Detterbeck FC, Wang Z and Loehrer PJ Sr: Standard outcome measures for thymic malignancies. J Thorac Oncol 5: 2017-2023, 2010

9. Kim ES, Putnam JB, Komaki R, Walsh GL, Ro JY, Shin HJ, Truong M, Moon H, Swisher SG, Fossella FV, et al: Phase II study of a multidisciplinary approach with induction chemotherapy, followed by surgical resection, radiation therapy, and consolidation chemotherapy for unresectable malignant thymomas: Final report. Lung Cancer 44: 369-379, 2004.

10. Rea F, Sartori F, Loy M, Calabrò F, Fornasiero A, Daniele O and Altavilla G: Chemotherapy and operation for invasive thymoma. J Thorac Cardiovasc Surg 106: 543-549, 1993.

11. Ströbel P, Marx A, Zettl A and Müller-Hermelink HK: Thymoma and thymic carcinoma: An update of the WHO classification 2004. Surg Today 35: 805-811, 2005 
12. Venuta F, Rendina EA, Pescarmona EO, De Giacomo T, Vegna ML, Fazi P, Flaishman I, Guarino E and Ricci C: Multimodality treatment of thymoma: A prospective study. Ann Thorac Surg 64: 1585-1592, 1997.

13. Gu ZT, Mao T, Chen WH and Fang W: Comparison of video-assisted thoracoscopic surgery and median sternotomy approaches for thymic tumor resections at a single institution. Surg Laparosc Endosc Percutan Tech 25: 47-51, 2015.

14. Toker A, Sonett J, Zielinski M, Rea F, Tomulescu V and Detterbeck FC: Standard terms, definitions, and policies for manimally invasive resection of thymoma. J Thorac Oncol 6 (7 Suppl 3): S1739-S1742, 2011.

15. Marulli G1, Lucchi M, Margaritora S, Cardillo G, Mussi A Cusumano G, Carleo F and Rea F: Surgical treatment of stage III thymic tumors: A multi-institutional review from four Italian centers. Eur J Cardiothorac Surg 39: e1-e7, 2011.

16. Jeong YJ, Lee KS, Kim J, Shim YM, Han J and Kwon OJ: Does CT of thymic epithelial tumors enable us to differentiate histologic subtypes and predict prognosis? AJR Am J Roentgenol 183: 283-289, 2004

17. Chen JL, Weisbrod GL and Herman SJ: Computed tomography and pathologic correlations of thymic lesions. J Thorac Imaging 3 : 61-65, 1988.

18. Fon GT, Bein ME, Mancuso AA, Keesey JC, Lupetin AR and Wong WS: Computed tomography of the anterior mediastinum in myasthenia gravis. A radiologic-pathologic correlative study. Radiology 142: 135-141, 1982.

19. Tomiyama N, Johkoh T, Mihara N,Honda O, Kozuka T, Koyama M, Hamada S, Okumura M, Ohta M, Eimoto T, et al: Using the world health organization classification of thymic epithelial neoplasms to describe CT findings. AJR Am J Roentgenol 179: 881-886, 2002.

20. Sadohara J, Fujimoto K, Müller NL, Kato S, Takamori S, Ohkuma K, Terasaki H and Hayabuchi N: Thymic epithelial tumors: Comparison of CT and MR imaging findings of low-risk thymomas, high-risk thymomas, and thymic carcinomas. Eur J Radiol 60: 70-79, 2006.

21. Yakushiji S, Tateishi U, Nagai S, Matsuno Y, Nakagawa K, Asamura $\mathrm{H}$ and Kusumoto M: Computed tomographic findings and prognosis in thymic epithelial tumor patients. J Comput Assist Tomogr 32: 799-805, 2008.
22. Marom EM, Milito MA, Moran CA, Liu P, Correa AM, Kim ES, Komaki R, Erasmus JJ, Hofstetter WL, Rice DC and Swisher SG: Computed tomography findings predicting invasiveness of thymoma. J Thorac Oncol 6: 1274-1281, 2011.

23. Priola AM, Priola SM, Di Franco M, Cataldi A, Durando S and Fava C: Computed tomography and thymoma: Distinctive findings in invasive and noninvasive thymoma and predictive features of recurrence. Radiol Med 115: 1-21, 2010 (In Italian).

24. Tomiyama N, Müller NL, Ellis SJ, Cleverley JR, Okumura M, Miyoshi S, Kusumoto M, Johkoh T, Yoshida S, Mihara N, et al: Invasive and noninvasive thymoma: Distinctive CT features. J Comput Assist Tomogr 25: 388-393, 2001.

25. Qu YJ, Liu GB, Shi HS, Liao MY, Yang GF and Tian ZX: Preoperative CT findings of thymoma are correlated with postoperative Masaoka clinical stage. Acad Radiol 20: 66-72, 2013.

26. Ruffini E, Filosso PL, Mossetti C, Bruna MC, Novero D, Lista P, Casadio $\mathrm{C}$ and Oliaro A: Thymoma: Inter-relationships among world health organization histology, Masaoka staging and myasthenia gravis and their independent prognostic significance: A single-centre experience. Eur J Cardiothorac Surg 40: 146-153, 2011.

27. Detterbeck FC, Nicholson AG, Kondo K, Van Schil P and Moran C: The Masaoka-Koga stage classification for thymic malignancies: Clarification and definition of terms. J Thorac Oncol 6 (7 Suppl 3): S1710-S1716, 2011

28. Tagawa T, Kometani T, Yamazaki K, Okamoto T, Wataya H, Seto T, Fukuyama S, Osoegawa A, Hirai F, Sugio K and Ichinose Y: Prognosis and therapeutic response according to the World Health Organization histological classification in advanced thymoma. Surg Today 41: 1599-1604, 2011.

29. Detterbeck FC, Moran C, Huang J, Suster S, Walsh G, Kaiser L and Wick M: Which way is up? Policies and procedures for surgeons and pathologists regarding resection specimens of thymic malignancy. J Thorac Oncol 6 (7 Suppl 3): S1730-S1738, 2011.

30. Zerhouni EA, Scott WW Jr, Baker RR, Wharam MD and Siegelman SS: Invasive thymomas: Diagnosis and evaluation by computed tomography. J Comput Assist Tomogr 6: 92-100, 1982. 